\title{
Nanoscale electrothermal co-simulation: compact dynamic models of hyperbolic heat transport and self-consistent device Monte Carlo
}

\author{
N. J. Pilgrim, W. Batty*, R. W. Kelsall and C. M. Snowden \\ Institute of Microwaves and Photonics, School of Electronic and Electrical Engineering, University of \\ Leeds, Leeds LS2 9JT, UK. \\ ${ }^{*}$ Corresponding author: \\ Tel. +44 $1133432089 \quad$ Fax +44 $1133437265 \quad$ E-mail w.batty@ee.leeds.ac.uk
}

\begin{abstract}
Two problems in the self-consistent, electrothermal co-simulation of nanoscale devices, are discussed. It is shown that the construction of dynamic compact thermal models for nanoscale devices, based on solution of the hyperbolic (wavelike) heat transport equation, can follow essentially the same approach as the authors' analytical thermal impedance matrix method for the parabolic (diffusive) equation. The analytical impedance matrix method for the time-independent case is employed in a thermally self-consistent device Monte Carlo simulation, illustrating the potential for detailed study of nanoscale electrothermal effects.
\end{abstract}

\section{INTRODUCTION}

It is recognised that with continuing progress in miniaturisation of device features, and development of structures characterised by nanometer length scales, that there is a growing demand for greater understanding of thermal transport in nanoscale devices. A number of problems arise in these small structures, for instance: inability of simple models to predict the thermal conductance of solid-solid interfaces; fundamental issues concerning the correct definitions of temperature in non-equilibrium nanosystems; lack of adequate models for phonon scattering rates in predictive simulations; lack of agreement between thermal models and experiment for semiconductor superlattices; and the modification of thermal transport by nearby interfaces and small heating volumes, with implications for thermal management [1]. Si microelectronics is increasingly characterised by deep sub-micron feature lengths, but microelectronic devices are still too large to be treated atomistically, so microscopic calculations of electronic and thermal transport must be based on solutions of the Boltzmann transport equation [1]. These solutions must capture both lattice self-heating effects, and local transport or hot carrier effects. For characteristic lengths shorter than $\sim 0.1 \mu \mathrm{m}$, and at high frequencies corresponding to gate switching on nanosecond or picosecond timescales, the model must also treat short-time transient thermal effects $[2]$.

Two such models are discussed in this paper: an approximate macroscopic model which goes be- yond Fourier's law and assumes that temperature waves are transmitted at acoustic speed and are attenuated due to relaxation [2]; and a detailed microscopic Monte Carlo model, with phonon transport described by the corresponding steady-state heat equation. It is first shown how compact dynamic thermal models, based on the hyperbolic heat transport equation, can be constructed in direct parallel with the authors' analytical thermal impedance matrix approach based on the parabolic heat equation. It is then shown how thermally selfconsistent Monte Carlo simulation can be used to provide a detailed microscopic description of device physics at the phonon level.

\section{COMPACT HYPERBOLIC SOLUTIONS}

The authors have presented previously, a detailed description of compact dynamic model construction based on solution of the classical heat diffusion equation [3]-[11]. This approach solved the equation,

$$
\nabla \cdot[\kappa(T) \nabla T]+g=\rho C \frac{\partial T}{\partial t}
$$

where $T$ is temperature, $t$ is time, $\kappa(T)$ is temperature dependent thermal conductivity, $g(x, y, z, t)$ is rate of heat generation, $\rho$ is density and $C$ is specific heat. This equation is non linear through the temperature dependence of $\kappa(T)$ (and possibly of $\rho$ and $C$ ). Transforming this equation to a linear form, and solving in Laplace transform $s$-space, the solution was expressed in the compact form,

$$
\overline{\Delta \theta_{i}}=\sum_{j} R_{T H_{i j}}(s) \overline{P_{j}}
$$

where $\overline{\Delta \theta_{i}}$ is the Laplace transformed temperature rise of element $i$ above its initial temperature, $R_{T H_{i j}}(s)$ is the thermal impedance matrix in Laplace $s$-space and the $\overline{P_{j}}$ are the transformed time-dependent fluxes due to power dissipation in elements, $j=1, \ldots, i, \ldots M$. As a simple illustration, for a heatsink mounted semiconductor die, with surface heating by active devices, $R_{T H_{i j}}(s)$ 
takes the simple form,

$$
\begin{aligned}
& R_{T H_{i j}}(s)=\frac{1}{\kappa_{S} L W} \times \\
& \sum_{m n} \frac{4 \tanh \left(\gamma_{m n} D\right)}{\gamma_{m n}\left(1+\delta_{m 0}\right)\left(1+\delta_{n 0}\right)} \frac{I_{m n}^{i} I_{m n}^{j}}{I_{00}^{i}}
\end{aligned}
$$

where $\kappa_{S}$ is thermal conductivity, $L, W, D$ are die $x, y, z$-dimension respectively, $\delta_{m n}$ is the Kronecker delta function, the $I_{m n}^{i}$ are double integrals over power dissipating regions, $D_{i}$, and

$$
\gamma_{m n}^{2}=\left(\frac{m \pi}{L}\right)^{2}+\left(\frac{n \pi}{W}\right)^{2}+\frac{s}{k_{S}}
$$

where $k_{S}$ is thermal diffusivity. Similar expressions hold for other thermal subsystems [5]. Performing the Laplace transformation, the time-domain step response, is

$$
\begin{aligned}
& R_{T H_{i j}}(t)=\mathcal{L}^{-1}\left\{R_{T H_{i j}}(s) \frac{1}{s}\right\} \\
& =\frac{1}{\kappa_{S} L W} \frac{2}{D} \sum_{l m n} \frac{4}{\left(1+\delta_{m 0}\right)\left(1+\delta_{n 0}\right)} \frac{I_{m n}^{i} I_{m n}^{j}}{I_{00}^{i}} \\
& \quad \times \frac{1-\exp \left\{-\pi^{2}\left[\left(\frac{l+1 / 2}{D}\right)^{2}+\left(\frac{m}{L}\right)^{2}+\left(\frac{n}{W}\right)^{2}\right] k t\right\}}{\pi^{2}\left[\left(\frac{l+1 / 2}{D}\right)^{2}+\left(\frac{m}{L}\right)^{2}+\left(\frac{n}{W}\right)^{2}\right]}
\end{aligned}
$$

with $l, m, n=0,1,2, \ldots$. It is shown in [5] how the steady-state part of this expression can be summed explicitly on $l$, and how the series can be accelerated.

The hyperbolic heat transport equation can be written [12],

$$
\nabla \cdot[\kappa(T) \nabla T]+g+\frac{\alpha}{\sigma^{2}} \frac{\partial g}{\partial t}=\rho C \frac{\partial T}{\partial t}+\frac{\kappa}{\sigma^{2}} \frac{\partial^{2} T}{\partial t^{2}}
$$

where $\sigma$ is the propagation speed for heat transfer and $\alpha / \sigma^{2}$ is the relaxation time for the heat flux to begin after a temperature gradient is imposed on the body. Solving this equation for the same illustrative system described above, the $s$-space thermal impedance matrix is obtained in identical form to Eq. 3, but now,

$$
\gamma_{m n}^{2}=\left(\frac{m \pi}{L}\right)^{2}+\left(\frac{n \pi}{W}\right)^{2}+\frac{s}{k_{S}}+\tau s^{2},
$$

with the additional term, $\tau s^{2}$. Performing the inverse Laplace transformation analytically [13], the time-domain step response takes the form,

$$
\begin{aligned}
& R_{T H_{i j}}(t)=\mathcal{L}^{-1}\left\{R_{T H_{i j}}(s) \frac{1}{s}\right\} \\
& \quad=\frac{1}{\kappa_{S} L W} \frac{2}{D} \sum_{l m n} \frac{4}{\left(1+\delta_{m 0}\right)\left(1+\delta_{n 0}\right)} \frac{I_{m n}^{i} I_{m n}^{j}}{I_{00}^{i}} \times
\end{aligned}
$$

$$
\begin{aligned}
& 1-\exp \left(\frac{-t}{2 \tau k_{s}}\right) \times \\
& \left\{\begin{array}{c}
\frac{1}{2 \tau k_{S}} \frac{\sin \left[\frac{t}{\tau^{1 / 2}}\left(\left(\frac{(l+1 / 2) \pi}{D}\right)^{2}+\left(\frac{m \pi}{L}\right)^{2}+\left(\frac{n \pi}{W}\right)^{2}-\frac{1}{4 \tau k_{S}^{2}}\right)\right]^{1 / 2}}{\left[\frac{1}{\tau^{1 / 2}}\left(\left(\frac{(l+1 / 2) \pi}{D}\right)^{2}+\left(\frac{m \pi}{L}\right)^{2}+\left(\frac{n \pi}{W}\right)^{2}-\frac{1}{4 \tau k_{S}^{2}}\right)\right]^{1 / 2}}+ \\
\cos \left[\frac{t}{\tau^{1 / 2}}\left(\left(\frac{(l+1 / 2) \pi}{D}\right)^{2}+\left(\frac{m \pi}{L}\right)^{2}+\left(\frac{n \pi}{W}\right)^{2}-\frac{1}{4 \tau k_{S}^{2}}\right)\right]^{1 / 2}
\end{array}\right\} \\
& \left(\frac{(l+1 / 2) \pi}{D}\right)^{2}+\left(\frac{m \pi}{L}\right)^{2}+\left(\frac{n \pi}{W}\right)^{2}-\frac{1}{4 \tau k_{S}^{2}}
\end{aligned}
$$

Comparing the above equation with the parabolic transient solution, it is apparent that the hyperbolic solution shows wavelike behaviour. The Laplace inverse can also be constructed numerically, so the whole of the authors' analytical thermal impedance matrix approach for the parabolic heat transport equation carries over almost trivially to the hyperbolic case.

\section{Monte CARlo transport modelling}

The term Monte Carlo is applied to a large range of mathematical techniques, typically those involving the use of distributions of stochastic variables. Such methods include Monte Carlo integration, applications in statistical physics and Monte Carlo transport models. The electronic modelling of charge carrier transport in semiconductor devices falls into the last of these categories.

In the standard Monte Carlo model in semiconductor physics, an ensemble of charge carriers are followed in time as they drift in local electric fields and scatter near-instantaneously off a range of scattering centres. Drift durations and scattering dynamics are determined stochastically, resulting in an intrinsic ability to simulate the physics of noise. Furthermore the use of a particle-based model requires no initial assumption on the form of the carrier distribution function. Through inclusion of multiple bands it is possible to extract a wide range of statistics including carrier velocities and energies, relative occupations of bands and distribution functions; transient parameters may also be extracted straightforwardly. Monte Carlo has been applied to both bulk systems and modelling of complex devices, where electronic self-consistency, heterostructures and other effects may be included.

At high temperatures, scattering arises primarily due to interaction with the lattice, which the Monte Carlo method is able to treat very accurately. Carrier-phonon scattering is split into a set of different processes, each causing differing electronic transitions and involving phonons from separate regions of the Brillouin zone (BZ). Each carrier-phonon scattering event results in a step increase or decrease in the related phonon population; through accumulation of these statistics a dynamic estimate of the microscopic heating rate may be determined, including the relative contri- 
butions of each type of scattering process. Since the state of each scattering carrier before and after an event is known, it is possible to localise the resultant step change in thermal energy not only by phonon branch but also in real- and reciprocalspace.

\section{A. Thermal flux in a 0.2 $\mu \mathrm{m}$ MESFET}

The authors have developed a Monte Carlo device simulator including electronic self-consistency through a two-dimensional Poisson equation solver and a standard 3-band spherical, non-parabolic model for the electronic bandstructure. This code has been applied to a $0.2 \mu \mathrm{m}$ gate MESFET with $0.2 \mu \mathrm{m}$ gate-source and gate-drain distances and a $5.10^{23} m^{-3} \mathrm{n}$-doped $0.1 \mu \mathrm{m}$-deep supply layer [14].

For the GaAs system, the main areas of the BZ which are perturbed through electron-phonon interaction are at the $\Gamma, L$ and $X$ points. The variation of power losses due to each set of phonons was examined by looking at the change with bias of total net phonon emission associated with $\Gamma, L$ and $X$ phonons. It was observed that at onset of saturation in the current-voltage characteristics the $\Gamma$ phonons dominate, with $X$ phonons becoming the primary power loss mechanism at around $4 \mathrm{~V}$ (gate bias is $0 \mathrm{~V}$, as with subsequent plots). Currently the sum of these powers are combined for use in the thermal solution; it is intended to investigate the effects of coupling between these phonons and the acoustic (thermally dissipating) phonons at a later stage.

The authors have also examined the spatial distribution of power emission, which results naturally from use of the Monte Carlo method. At high source-drain biases the great majority of power losses are located just within the drain contact edge (Fig. 1; bottom). For lower biases the heating in the channel is visible, as seen in the top of Fig. 1. Moglestue et al. [15] have examined thermal losses in this way, with a view to examining device degradation, but the authors know of no further work on this topic.

\section{B. Thermally self-consistent Monte Carlo}

In addition to the extraction of thermal power the authors have also considered the use of this data in construction of thermally self-consistent Monte Carlo results. Due to the diverse electronic and thermal timescales initially focus has been placed on calculation of steady-state characteristics using a leapfrog algorithm, alternating between solution of the electronic (Monte Carlo) and thermal problems. The authors are aware of only a few attempts at electrothermal Monte Carlo modelling [16]-[18], each involving application to different devices and with a range of approximations to the electronic and thermal systems; the authors' system is described briefly below.

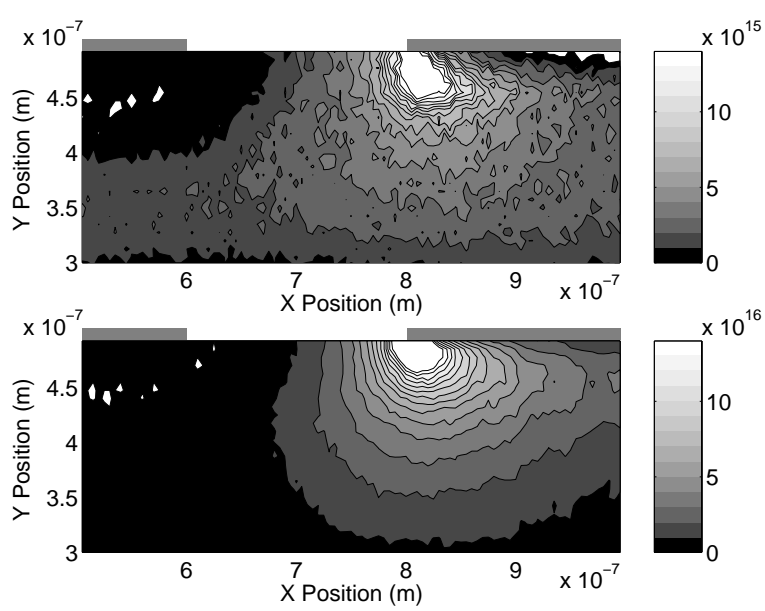

Fig. 1. Spatial distribution of net phonon emission at $V_{d s}$ of $1 \mathrm{~V}$ (upper) and $5 \mathrm{~V}$ (lower).

Initially results are dgenerate for the $0.2 \mu \mathrm{m}$ MESFET as above; for the thermal domain we choose a GaAs die of $500 \times 500 \times 125 \mu \mathrm{m}$ with the device centred at the top. The thermal solution is currently two-dimensional, with thermal power assumed to be generated uniformly over the full $500 \mu \mathrm{m}$ width and dissipated through a heatsink over the base of the die held at an ambient $300 \mathrm{~K}$. This thermal description utilises the authors' fully analytical thermal impedance matrix approach [5], applied to the time-independent heat diffusion equation, and is readily generalised to the 3-dimensional case, or to treat detailed device structure, such as surface metallisation. This analytical impedance matrix method avoids the need for temperature solution in areas where this is not required for use in subsequent Monte Carlo iterations, i.e., far from the device active region, yet still includes the large-scale thermal boundary conditions. Thermal nonlinearity is included through application of the Kirchhoff transformation [5].

In the initial Monte Carlo iteration the temperature profile is assumed to be at the ambient $300 \mathrm{~K}$; in subsequent iterations the non-uniform temperature profiles generated are integrated into the Monte Carlo model through a stochastic rejection technique [19]. This involves calculation of the electron-phonon scattering rates using the maximal phonon occupation number, with an associated probability that a given scattering event may be rejected depending upon the ratio of the local and maximal occupations:

$$
P_{\text {real }}=\frac{N_{\text {real }}+\beta}{N_{\text {max }}+\beta},
$$

where $\beta$ is unity for emission processes and zero for absorption processes.

Results when including thermal self-consistency show the characteristic 'thermal droop' in the 
source-drain currents, which can be seen in Fig. 2 for a $0 \mathrm{~V}$ (applied) gate bias. The peak temperature in the device along the same biases (Fig. 3) shows a strongly linear rise after current saturation is achieved, which is similar to a result obtained by Yoder and Fichtner [17].

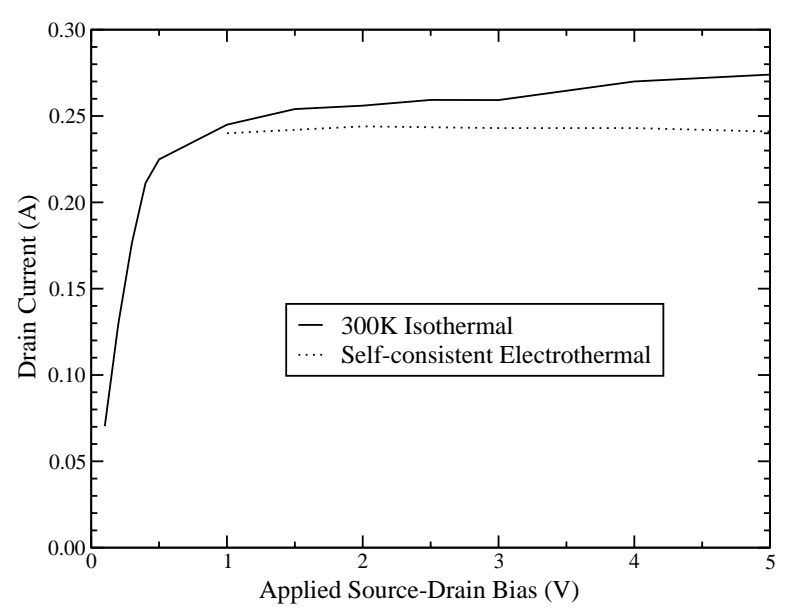

Fig. 2. MESFET I-V characteristics, with and without thermal consistency, at an applied gate of $0 \mathrm{~V}$.

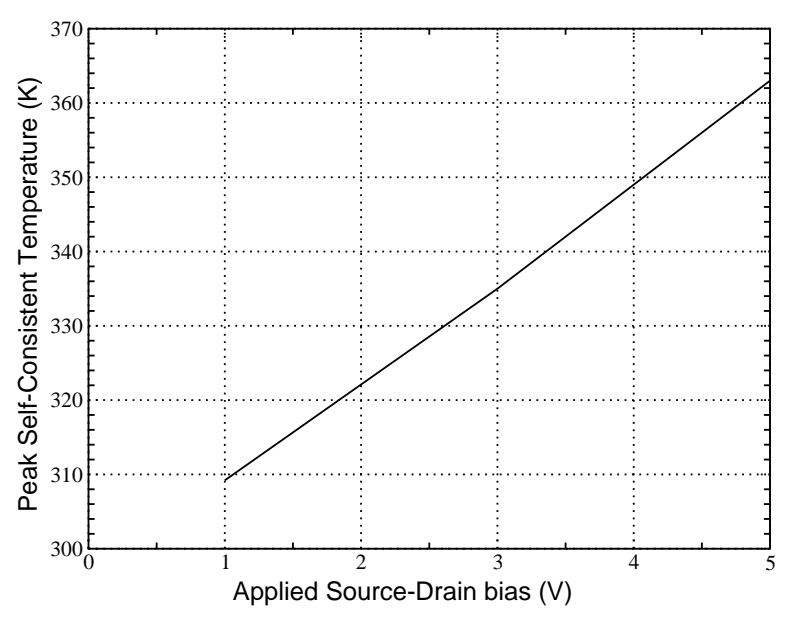

Fig. 3. Variation of peak temperature within the MESFET over the same biases as in figure 2 .

\section{Conclusion}

It has been demonstrated that the authors' analytical thermal impedance matrix approach can be applied straightforwardly to wavelike thermal solutions based on the hyperbolic heat conduction equation. Dynamic compact thermal models can therefore readily be constructed for very smal devices and high frequency operation. The thermal impedance matrix approach has been applied to the steady-state, self-consistent electrothermal Monte Carlo simulation of a nanoscale device. These simulations illustrate the potential of Monte Carlo simulation for detailed microscopic study of thermal physics. Future work will couple the timedependent thermal impedance matrix solution to the Monte Carlo simulation, for self-consistent treatment of the transient electrothermal problem.

\section{ACKNOWLEDGEMENT}

This work was supported by the U.S. Army Research Office through Clemson University as a Multidisciplinary Research Initiative on Quasi-Optics, agreement Number DAAG55-97-K-0132.

\section{REFERENCES}

[1] D. G. Cahill, W. K. Ford, K. E. Goodson, G. D. Mahan, A. Majumdar, H. J. Maris, R. Merlin and S. R. Phillpot, "Nanoscale thermal transport," J. Appl. Phys., Vol. 93, No. 2, pp. 793-818, Jan. 2003.

[2] O. J. Ilegbusi, A. U. Coskun and Y. Yener, "Application of spectral methods to thermal analysis of nanoscale electronic devices," Num. Heat Transfer, Part A, Vol. 41, pp. 711-724, 2002.

[3] W. Batty, A. J. Panks, R. G. Johnson and C. M. Snowden, "Electro-thermal modeling and measurement for spatial power combining at millimeter wavelengths," IEEE Trans. Microwave Theory Tech., Vol. 47, No. 12, pp. 2574-2585, 1999.

[4] W. Batty, A. J. Panks, R. G. Johnson and C. M. Snowden, "Electro-thermal modeling of monolithic and hybrid microwave and millimeter wave IC's," VLSI Design, Vol. 10, No. 4, pp. 355-389, 2000.

[5] W. Batty, C. E. Christoffersen, A. J. Panks, S. David, C. M. Snowden and M. B. Steer, "Electro-thermal CAD of power devices and circuits with fully physical timedependent compact thermal modeling of complex nonlinear 3-d systems," IEEE Trans. Comp. Packag. Technol., Vol. 24, No. 4, pp. 566-590, Dec. 2001.

[6] W. Batty, C. E. Christoffersen, A. B. Yakovlev, J. F. Whitaker, A. Mortazawi, A. Al-Zayed, M. Ozkar, S. C. Ortiz, R. M. Reano, K. Yang, L. P. B. Katehi, C. M. Snowden and M. B. Steer, "Global coupled EMelectrical-thermal simulation and experimental validation for a spatial power combining MMIC array," IEEE Trans. Microwave Theory Tech., Vol. 50, No. 12, pp. 2820-2833, Dec. 2002.

[7] W. Batty and C. M. Snowden, "Electro-thermal device and circuit simulation with thermal nonlinearity due to temperature dependent diffusivity," Electron. Lett., Vol. 36, pp. 1966-1968, 2000.

[8] K. Krabbenhoft and L. Damkilde, "Comment: Electrothermal device and circuit simulation with thermal nonlinearity due to temperature dependent diffusivity," Electron. Lett., Vol. 37, pp. 1481-1482, 2001.

[9] W. Batty, S. David and C. M. Snowden, "Reply to Comment on 'Electro-Thermal Device and Circuit Simulation with Thermal Non Linearity due to Temperature Dependent Diffusivity'," Electron. Lett., Vol. 37, pp. 1482-1483, 2001.

[10] W. Batty, S. David, A. J. Panks, R. G. Johnson and C. M. Snowden, "Series acceleration of a compact thermal model and fast nonlinear optimization of electrothermal device design," Proc. 7th Internat. Workshop on Thermal Investigations of ICs and Systems (THERMINIC 2001), Paris, pp. 11-16, Sept. 2001.

[11] W. Batty, A. J. Panks, C. E. Christoffersen, S. David, R. G. Johnson, C. M. Snowden and M. B. Steer, "Fully analytical compact thermal model of complex electronic power devices and packages in coupled electrothermal CAD," Proc. 7th Internat. Workshop on Thermal Investigations of ICs and Systems (THERMINIC 2001), Paris, pp. 99-102, Sept. 2001.

[12] J. V. Beck, K. D. Cole, A. Haji-Sheikh and B. Litkouhi, "Heat Conduction Using Green's Functions," Hemisphere Publishing Corporation, 1992.

[13] F. Oberhettinger and L. Badii, "Tables of Laplace Transforms," Springer-Verlag, 1973. 
[14] N. J. pilgrim, W. Batty and R. W. Kelsall, "Thermally self-consistent Monte Carlo device simulations," J. Comp. Electron., Vol. 1, pp. 263-266, 2002.

[15] C. Moglestue, F. A. Buot and W. T. Anderson, "Ensemble Monte Carlo particle investigation of hot electron induced source-drain burnout characteristics of GaAs field-effect transistors," J. Appl. Phys., Vol. 78, No. 4, pp. 2343-2348, 1995.

[16] K. Tarnay. A. Gali, A. Poppe, T. Kocsis and F. Masszi. "Examination of MOS structures by a 3D particle dynamics Monte-Carlo simulator including electrothermal effects," Physica Scripta, Vol. T69, pp. 290-294, 1997.

[17] P. D. Yoder and W. Fichtner, "Effects of scaling and lattice heating on n-MOSFET performance via electrothermal Monte Carlo simulation," Proc. Simulation of Semiconductor Devices and Processes, Eds. K. de Meyer and S. Biesemans, pp. 165-168, 1998.

[18] R. P. Joshi, S. Pathak and J. A. Mcadoo, "Hot-electron and thermal effects on the dynamic characteristics of single-transit $\mathrm{SiC}$ impact-ionization avalanche transittime diodes," J. Appl. Phys., Vol. 78, No. 5, pp. 34923497, 1995.

[19] C. Jacoboni and L. Reggiani, "The Monte Carlo method for the solution of charge transport in semiconductors with applications to covalent materials," Rev. Mod. Phys., Vol. 55, No. 3, pp. 645-705, July 1983. 